RECYT

Año 23 / No 35 / 2021 / 44-53

\title{
Una revisión de los sistemas recomendadores grupales como herramienta innovadora en el área del turismo
}

\section{A survey on group recommender systems as an innovative tool in the tourism area}

\author{
${ }^{1}$ Universidad de Holguín, Holguín, Cuba. \\ ${ }^{2}$ Hotel Pullman Cayo Coco, Ciego de Ávila, Cuba. \\ 3universidad de Ciego de Ávila, Ciego de Ávila, Cuba. \\ *E-mail: ryera@unica.cu \\ Recibido: 05/02/2021; Aprobado 15/04/2021
}

Yilena Pérez-Almaguer ${ }^{1}$, Neober Martín-Dueñas² ${ }^{2}$ Edianny Carballo-Cruz ${ }^{3}$, Raciel Yera ${ }^{3,{ }^{*}}$

\section{Resumen}

Los sistemas recomendadores son herramientas enfocadas en ayudar a los usuarios a obtener la información que mejor se corresponde con sus intereses y preferencias en un espacio de búsqueda sobrecargado de posibles opciones. A su vez, los sistemas recomendadores grupales se centran en la sugerencia de determinados tipos de ítems que tienden a ser consumidos en grupos. El presente trabajo se centra en realizar un análisis de la utilización de los sistemas recomendadores grupales como herramienta innovadora en el área del turismo, la cual constituye una de las áreas de aplicación más importantes de los sistemas recomendadores. Específicamente, se lleva a cabo un estudio de los traba os más recientes en esta línea de investigación, realizándose una comparación atendiendo a tipo de estudio, tipo de evaluación, escenario de aplicación, fortalezas, debilidades y país en que se desarrolla el estudio. Esta comparación dio lugar a la proyección de líneas futuras entre las que se destaca el desarrollo de investigaciones de ciclo completo que incluyan nuevos algoritmos y metodologías que concluyan con un extenso estudio experimental que garantice una apropiada reutilización de los métodos propuestos.

Palabras clave: Sistemas Recomendadores Grupales; Turismo; Preferencias; Revisión; Comparación.

\section{Abstract}

Recommender systems are tools focused on helping users to get the information that best fits their preferences and needs in a search space overloaded with possible alternatives. In addition, group recommender systems are centered on suggesting certain kind of items that tend to be consumed in groups. The current research work is focused on performing an analysis of the use of group recommender systems as an innovative tool in the tourism area, which is one of the most relevant application areas in recommender systems. Specifically, it is performed a study of the most recent research works in this area, doing a comparison among them according to kind of study, kind of evaluation, application scenario, strengths, weaknesses, and country. This comparison leads to the projection of future research lines such as the development of new algorithms and methodologies that concludes with an extensive experimental study that assures the appropriate reuse of the proposed methods.

Keywords: Group Recommender Systems; Tourism; Preferences; Survey; Comparison.

\section{Introducción}

Los sistemas recomendadores son herramientas enfocadas a ayudar a los usuarios a obtener aquella información que mejor se corresponda con sus intereses y preferencias. Mientras que un buscador habitual se centra en encontrar aquello que el usuario solicita, un sistema recomendador ayuda al usuario a tomar una decisión, que puede ser la compra de un producto en un portal de comercio electrónico, la lectura de un libro, la revisión de un artículo científico, el acceso a una página web en específico, o el estudio de determinado recurso educativo en una platafor- ma virtual de aprendizaje.

La clasificación más popular de los sistemas recomendadores está asociada al algoritmo que emplean para realizar la tarea de minería correspondiente y divide a los métodos de recomendación en métodos de filtrado basado en el contenido, métodos de filtrado colaborativo, métodos de filtrado demográfico y métodos híbridos [14]. En adición, la literatura ha desarrollado tanto sistemas recomendadores para la sugerencia de ítems para usuarios individuales, como enfocados en grupos de usuarios [5]. Así, los sistemas recomendadores enfocados en grupos de usuarios se centran en la sugerencia de determinados tipos 
de ítems que tienden a ser consumidos en grupos y no por usuarios individuales, tales como programas de televisión y paquetes turísticos [6].

De manera general, los dominios iniciales de aplicación de los sistemas recomendadores han sido el e-commerce [7] y el e-learning [8,9], aunque en los últimos tiempos estos sistemas están siendo aplicados a escenarios cada vez más diversos [10-12]. Así, son relevantes las aplicaciones de los sistemas recomendadores en escenarios de $e$-health [13] y de e-tourism [14], como dos contextos relevantes de particular importancia.

Específicamente, resulta importante en los últimos años el desarrollo de sistemas recomendadores en el dominio del turismo [10]. En este dominio existe mucha información en línea disponible y por tanto los sistemas recomendadores juegan un papel muy importante con vistas a ayudar a los usuarios en la toma de decisiones sobre qué paquete turístico comprar, qué instalación hotelera visitar, o qué recorrido turístico elegir, entre otras decisiones similares a tomar con vistas a lograr la satisfacción final del cliente [15].

En este sentido, a pesar de ser un área con bastante desarrollo $[16,17]$, se ha identificado en la literatura una ausencia de trabajos de revisión que realicen un análisis global de dichos avances en el caso particular del desarrollo de sistemas recomendadores grupales en el área del turismo. Lo anterior sirve como motivación para el desarrollo del presente trabajo, el cual tiene como principales contribuciones las siguientes:

- El desarrollo de una revisión detallada de las contribuciones enfocadas en sistemas recomendadores grupales en el área del turismo.

- El desarrollo de una comparación entre los trabajos identificados, resaltando las fortalezas y debilidades en cada caso.

- Un análisis de líneas futuras de trabajo a seguir en esta área de investigación, considerando el contexto contemporáneo de innovación en el que se desarrolla la industria del turismo.

El resto del trabajo se estructura de la siguiente manera. La sección 2 presenta varios elementos básicos sobre sistemas recomendadores, esenciales para la comprensión del trabajo. Esto incluye el principio de funcionamiento y clasificación de los sistemas recomendadores, aplicaciones de los sistemas recomendadores en el turismo y fundamentos de recomendación grupal. La sección 3 presenta el desarrollo de la revisión, incluyendo metodología y análisis de los trabajos. La sección 4 presenta la discusión de los resultados, realizando una comparación entre los trabajos identificados, atendiendo a tipo de estudio, tipo de evaluación, escenario de aplicación, fortalezas, debilidades y país en que se desarrolla el estudio. En adición, se proyectan las líneas futuras a desarrollar. El trabajo finaliza con la sección de conclusiones.

\section{Elementos básicos sobre sistemas recomendadores}

La presente sección realiza un estudio de los elementos básicos sobre los sistemas recomendadores, tales como el principio de funcionamiento y clasificación de los sistemas recomendadores, aplicaciones de los sistemas recomendadores en el turismo, y fundamentos de recomendación grupal.

\section{Principio de funcionamiento y clasificación de los sistemas recomendadores}

La figura 1 muestra un esquema del funcionamiento básico de un sistema recomendador, en el que inicialmente un usuario necesita elegir qué ítems consumir dentro de un gran conjunto, y para esto le solicita sugerencias al sistema (1). Tras esto, el recomendador procesa la información asociada a los ítems y a todos los perfiles de usuario (incluyendo el usuario actual) (2, 3), para posteriormente sugerir los ítems más apropiados (4), quedando el usuario libre de realizar la elección que él desee, basándose en estas recomendaciones (5).

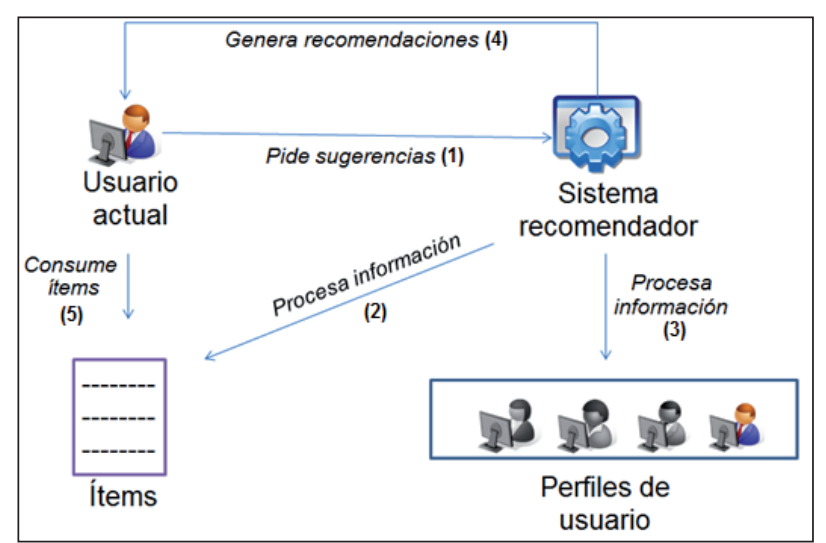

Figura 1: Esquema del funcionamiento de un sistema recomendador.

Los recomendadores basados en el contenido [18] recomiendan basándose exclusivamente en la descripción del ítem y de un perfil con los intereses del usuario activo, teniendo en cuenta la filosofía de "recomiéndame las cosas que he seleccionado o me han gustado anteriormente". Estos sistemas se caracterizan por contener un modelo que describe los ítems que pueden ser recomendados, un modo de crear un perfil de usuario que representa los tipos de ítems que este prefiere, y un modo de comparar cada uno de los ítems a recomendar con el perfil del usuario, para determinar cuáles de ellos serán recomendados.

Los ítems a ser recomendados suelen caracterizarse a través de un conjunto de rasgos o atributos, conociéndose los valores que pueden tomar cada uno de estos atributos. Considerando el grado de preferencia de un usuario por un subconjunto de ítems y utilizando algoritmos de aprendizaje, es posible construir su perfil en términos de los mismos rasgos; y a partir de este, obtener de los ítems restantes 
aquellos de mayor utilidad para el referido usuario. Las dos cuestiones más importantes en estos sistemas son, por tanto, la representación de los ítems y el aprendizaje del perfil del usuario.

Los recomendadores basados en filtrado colaborativo $[19,20]$, por otra parte, inicialmente permiten a los usuarios proporcionar valores de preferencias personales acerca de un conjunto de objetos (videos, canciones, filmes), de forma tal que una vez que haya suficiente información almacenada en el sistema, sea posible realizar recomendaciones basándose en la información proporcionada por aquellos usuarios con un comportamiento semejante al del usuario actual. De forma alternativa, las preferencias de los usuarios también pueden ser adquiridas implícitamente.

Los sistemas recomendadores demográficos [21, 22] tienen como objetivo clasificar al usuario según sus atributos personales y hacer las recomendaciones basándose en sus clases demográficas. El funcionamiento de estos está justificado bajo el principio de que individuos con ciertos atributos personales en común (sexo, edad, país), también deben tener preferencias comunes.

Finalmente, los sistemas recomendadores híbridos [23] frecuentemente usan una combinación de las técnicas anteriores con vistas a explotar los méritos de cada una de ellas por separado. Así, se han desarrollado enfoques que combinan filtrado colaborativo con información demográfica [24], enfoques de filtrado colaborativo con métodos basados en el contenido [25], y enfoques que combinan diferentes técnicas de filtrado colaborativo [26]. Burke [23] menciona siete formas básicas en las que se pueden combinar varios métodos de recomendación, con vistas a construir métodos híbridos. Estas son las basadas en mezcla, en pesos, en intercambio, en cascada, en combinación de características, en aumento de características $\mathrm{y}$ en meta-nivel.

\section{Aplicaciones de los sistemas recomendadores en el turismo}

Numerosas han sido en los últimos años las aplicaciones en el área de los sistemas recomendadores. Lu et al. [10] han realizado una revisión de las principales áreas de aplicación, identificando ocho grandes grupos de trabajos, que son e-government, e-business, e-commerce, e-library, e-learning, e-tourism, e-resources y e-group activity.

En el caso particular del turismo electrónico o $e$ tourism, Chaudhari y Thakkar [17] y Renjith et al. [16] han recientemente elaborado una revisión de los trabajos disponibles en el estado del arte de esta área de investigación. Entre las categorías identificadas para agrupar a los trabajos realizados, se encuentran los sistemas recomendadores de hoteles [27]; los sistemas recomendadores de restaurantes [28]; los sistemas recomendadores de paquetes turísticos que incluyen planeación de rutas y paquetes de viajes [29]; y los sistemas recomendadores de atracciones puntuales como puntos de interés y museos [30].

A pesar de identificarse un importante grupo de trabajos en estas recientes revisiones, resulta necesario resaltar que el grueso de estos trabajos se centra en el desarrollo de sistemas recomendadores individuales. Se lleva a cabo tanto en estos recientes trabajos de revisión como en la literatura en general, un análisis muy limitado de trabajos centrados en la recomendación grupal. Esto se contrapone con la naturaleza de muchos productos turísticos, los cuales son consumidos en grupo.

A raíz de lo anterior, el presente trabajo lleva a cabo un análisis de los trabajos más recientes enfocados en la tarea de la recomendación grupal en el dominio del turismo.

\section{Fundamentos de la recomendación grupal}

En la presente sección se realiza un breve análisis de los fundamentos de la recomendación grupal, el cual resulta necesario en el contexto del presente trabajo.

Los sistemas recomendadores grupales han ganado en popularidad en los últimos años $[5,6]$, considerando que existe un grupo de ítems tales como programas de televisión y paquetes turísticos, que poseen una tendencia a ser consumidos por grupos y no por usuarios individuales. En este escenario, la recomendación de ítems que satisfagan las preferencias del grupo, puede llegar a ser una tarea compleja en comparación con la de la recomendación individual.

Con vistas a cumplir su propósito, los sistemas recomendadores grupales usualmente se construyen como una extensión de los sistemas recomendadores individuales, apoyándose en la agregación de la información asociada a cada usuario individual. Los dos enfoques principales de recomendación grupal abordados por la literatura son:

- La agregación de ratings, la cual crea el perfil de un pseudo-usuario que combina las preferencias del grupo. Este perfil es usado como el receptor final de las recomendaciones generadas, con vistas a satisfacer las preferencias globales del grupo [5, 31].

- La agregación de recomendaciones, la que se basa en la agregación de las recomendaciones generadas de manera individual a cada uno de los miembros del grupo, con vistas a obtener la lista global de recomendaciones $[5,31]$.

\section{Desarrollo de la revisión}

La presente sección desarrolla la revisión de los sistemas recomendadores grupales como herramienta innovadora en el área del turismo. Inicialmente se presenta la metodología llevada a cabo en el proceso de revisión. En un segundo momento, se realiza el análisis de los trabajos identificados. 


\section{Metodología}

En la presente sección se muestra la metodología a seguir para la revisión de los trabajos recientes en sistemas recomendadores grupales aplicados al turismo, acorde a otras revisiones anteriores en otras líneas de trabajo en sistemas recomendadores [32].

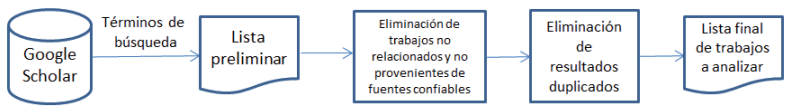

Figura 2: Metodología a seguir durante el proceso de revisión.

La figura 2 presenta un esbozo de esta metodología, la cual se compone de los siguientes pasos:

-Obtención del listado inicial de posibles trabajos a analizar, a partir de Google Scholar. Con este fin, se utiliza como cadena de búsqueda "group recommender systems tourism", analizándose los 100 primeros trabajos devueltos, desde 2016 hasta la fecha (Diciembre 2020). En este sentido, se considera interesante el estudio de la tendencia de la investigación en esta línea en estos últimos cinco años, tomando en cuenta que para etapas previas se ha reportado poco desarrollo en esta dirección [31, 33]. Asimismo, si bien se identificó un número de trabajos más allá de estos primeros 100 trabajos, se detectó que por encima de esta cifra los resultados obtenidos se alejaban del propósito de esta revisión, y por tanto no fueron considerados para un análisis más profundo.

-Eliminación de trabajos no relacionados y no provenientes de fuentes confiables. Aquí se dejaron de considerar del listado inicial, algunos trabajos que no abordan el problema de la recomendación grupal en turismo a pesar de ser obtenido con la cadena de búsqueda utilizada. En adición, se dejaron de considerar trabajos que no cumplían con criterios de calidad como la indización en bases de datos reconocidas como Web of Science, Scopus, Scielo; o publicados por editoriales reconocidas en el área de las ciencias de la computación, como Elsevier, Springer, Wiley, o IEEE.

-Eliminación de resultados duplicados, considerando que en varios casos se detectó la publicación de varios trabajos asociados a un mismo resultado. En este escenario, se dejaron de considerar trabajos centrados en presentar resultados parciales de investigación, resultados que también forman parte de otros trabajos de mayor extensión sí incluidos en esta revisión.

Una vez llevado a cabo este proceso, se obtuvo un listado final de trabajos a analizar, conformado por 8 trabajos.

\section{Análisis de los trabajos}

En la presente sección se realiza un análisis detallado de los trabajos centrados en recomendación grupal para el área del turismo, identificados en la sección anterior.

\section{Sistema recomendador grupal basado en relaciones sociales}

Christensen et al. [34] desarrollan un sistema recomendador grupal para el dominio del turismo, que se basa en la construcción de un perfil del grupo que incorpora las relaciones sociales entre los miembros del grupo, en adición a las preferencias de estos. En adición, la generación de las recomendaciones individuales y grupales se realiza a través de una técnica híbrida que combina filtrado colaborativo, basado en contenido y demográfico.

Específicamente, las recomendaciones grupales (Figura 3) son generadas usando las preferencias del grupo, los perfiles individuales de cada miembro y las relaciones entre dichos miembros. En este trabajo, las relaciones se representan a través de una ponderación en dependencia de la categoría de esta, considerándose categorías como padre/madre, pareja, hijo/hija, amigo, colega de trabajo, etc. Dicha ponderación es utilizada en la generación del listado de recomendaciones finales.

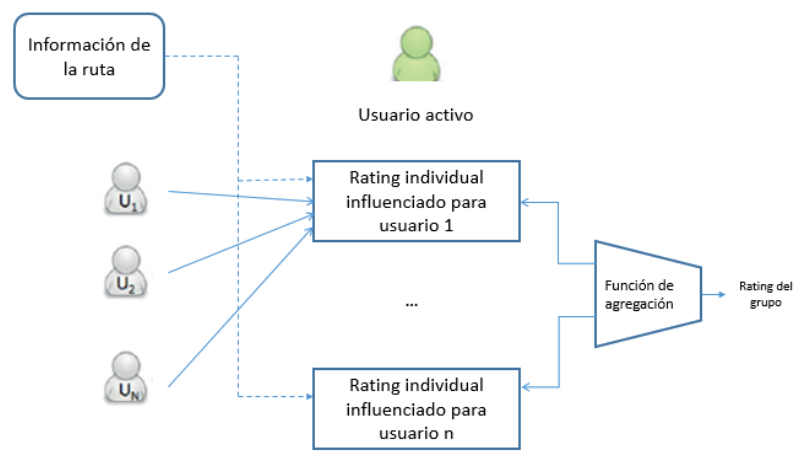

Figura 3: Funcionamiento del sistema recomendador grupal basado en relaciones sociales.

La evaluación de la propuesta se realiza a través del desarrollo de un sistema recomendador de rutas turísticas, denominado Hermes, que incluye recomendación individual y grupal. Aquí se llevó a cabo un conjunto de experimentos sobre este sistema, utilizando una base de datos creada artificialmente y compuesta por 1300 rutas y 800 usuarios. Sobre esta base de datos, se realizó la comparación entre la propuesta y otros métodos previamente desarrollados, utilizando métricas tradicionales como MAE y Precision [35]. Se muestra que la propuesta da lugar a una mejora en la eficacia de la recomendación, tanto a nivel individual como grupal.

\section{Sistema recomendador basado en la contribución de cada miembro del grupo}

Wang et al. [36] presentan un enfoque de recomendación grupal de propósito general, que tiene como meta la generación de recomendaciones tomando en consideración la contribución de cada miembro del grupo acorde a su grado de importancia. Este grado de importancia de cada 
miembro es obtenido a través de una técnica de factorización matricial.

El desarrollo de un método de recomendación que tiene en cuenta este grado de importancia, posibilita una modelación del grupo mucho más precisa en escenarios donde la matriz de ratings es altamente incompleta y dispersa. En adición, también se propone un método de recomendación basado en la distancia Manhattan, que permite capturar una aproximación local de las preferencias promedio del grupo, y que permite mejorar también la eficacia de la recomendación. Ambos métodos se integran en un único enfoque que está compuesto por dos etapas que respectivamente se corresponden por cada uno de los dos mencionados métodos.

La propuesta se evalúa utilizando bases de datos internacionales de referencia en el área de los sistemas recomendadores, como Movielens y Jester. En adición, se presenta un sistema recomendador de actividades turísticas, donde se muestra la aplicación de la propuesta en este escenario. No obstante, este sistema no constituye un elemento central en el desarrollo de esta investigación.

\section{Sistema recomendador híbrido de servicios de viajes}

De Pessemier et al. [37] proponen un sistema recomendador que ofrece sugerencias personalizadas de destinos turísticos a usuarios individuales y a grupos. En el caso particular de los grupos, la recomendación se lleva a cabo en dos momentos. En un primer momento, las recomendaciones generadas a cada individuo particular, son combinadas utilizando una técnica de agregación de recomendaciones con vistas a conformar la recomendación grupal.

En un segundo momento, el sistema actúa como un recomendador conversacional, en el cual cada miembro tiene la posibilidad de establecer un ranking de sus preferencias acorde a las recomendaciones grupales candidatas. Estos rankings son procesados a través de la regla Borda [38], para finalmente obtener las elecciones ganadoras.

Como parte del trabajo también se propone el método de recomendación individual a utilizar, que se basa en una combinación de recomendación basada en contenido, basada en filtrado colaborativo y basada en el conocimiento. Concretamente, el método se construye como un enfoque híbrido, donde se mezclan las salidas individuales de los tres enfoques de recomendación mencionados.

La evaluación de la propuesta se realiza en tres fases. Una primera fase evalúa la calidad general del prototipo de sistema recomendador de viajes construido, para lo cual se realiza un cuestionario a los usuarios. Una segunda fase, evalúa la percepción de los usuarios sobre la calidad independiente de cada enfoque utilizado (basado en contenido, de filtrado colaborativo y basado en contenido). Finalmente, una tercera fase realiza la evaluación de la recomendación grupal, también a través de un cuestionario con grupos y usuarios reales.

\section{Sistema recomendador soportado por un chat, en el dominio del turismo}

Nguyen y Ricci [27] ilustran un nuevo enfoque para la recomendación grupal en el dominio del turismo, que toma en cuenta la dinámica de las interacciones de los individuos en el contexto real del grupo. Este enfoque se desarrolla a través de un sistema montado sobre dispositivos móviles, que monitorea y explota las interacciones de los usuarios en un grupo de discusión, y ofrece recomendaciones apropiadas así como otros tipos de sugerencias para guiar y ayudar a los miembros del grupo con vistas a llegar a un acuerdo.

La lógica de la recomendación implica la observación de las acciones de los usuarios durante el grupo de discusión, para inferir información del usuario sobre sus preferencias en el contexto específico del grupo. Esto se realiza a través del cálculo de una ponderación de cada una de las características de los ítems a recomendar, con respecto al usuario activo. Esta ponderación es subsecuentemente utilizada para calcular la utilidad de cada ítem para dicho usuario. A su vez, esta información es posteriormente utilizada para calcular los vectores de ponderación del grupo, mediante una agregación de los vectores de los usuarios en la que se realiza un promedio pesado y se considera también el rol de cada miembro del grupo.

La evaluación de la propuesta se realiza mediante un estudio de usuario centrado en medir la usabilidad de esta, la calidad percibida de las recomendaciones grupales y la satisfacción de la opción elegida por el grupo.

\section{Recomendación turística soportada por secuenciación de puntos de interés}

Herzog et al. [39] presentan TourRec, un sistema recomendador de rutas turísticas para individuos y grupos, en el que las rutas turísticas se definen como una secuencia de puntos de interés. Antes de utilizar el sistema, el usuario tiene la opción de evaluar diferentes categorías de puntos de interés, tales como "Arte y Entretenimiento", "Alimentación”, etc, en un rango de 0 a 5 . Tras esto, el turista puede solicitar una sugerencia de ruta especificando un origen, un destino, la hora de inicio y la duración máxima del viaje. El sistema colecta puntos de interés en las proximidades de los orígenes y destinos, les asocia una valoración acorde a las preferencias del usuario y el contexto, y los combina posteriormente hasta obtener una ruta agradable para el usuario. En adición, se estudiaron varios algoritmos para la generación del viaje, tales como extensiones del algoritmo de Dijkstra [39].

Este esquema se entiende para las recomendaciones grupales, obteniendo estas a través de la agregación de los modelos de los usuarios o de las recomendaciones individuales. Aquí se emplearon varios enfoques tradicionales de agregación como el promedio o la estrategia 
del máximo. En adición se consideraron otras estrategias de agregación como agregar solo una parte de las rutas obtenidas individualmente, o fraccionar el grupo en partes específicas de estas.

La evaluación de la propuesta se realiza a través de un sistema real, con usuarios reales, construido con este fin.

\section{Recomendador soportada por discusión y negociación de las preferencias}

Álvarez y Ziegler [40] presentan un nuevo enfoque para la recomendación grupal que se centra en la interacción social entre los miembros del grupo, durante la formulación, discusión y negociación de las características que los ítems conjuntos a ser seleccionados deben poseer.

De manera particular, se proponen tres enfoques para llevar a cabo este proceso, en cuya construcción se toma en consideración el nivel sobre el cual los usuarios pueden establecer sus preferencias (nivel individual o nivel grupo), y el tipo de información sobre la cual se necesita consenso entre los usuarios, con vistas a ser finalmente considerada para la generación de recomendaciones.

El trabajo explora los posibles beneficios de este enfoque a través del desarrollo de tres prototipos de aplicaciones en el dominio de la recomendación de viajes, las que son evaluadas con grupos de usuarios de diferente tamaño. Aquí mide cuestiones relacionadas con la efectividad de los diferentes métodos para compartir la información en el grupo, y la repercusión del tamaño del grupo en el proceso de recomendación.

\section{Recomendación de viajes apoyada por geolocalización}

Longesh et al. [41] presentan un sistema recomendador de viajes que se apoya en redes sociales basadas en geolocalización, centradas en compartir los puntos de interés visitados por los usuarios. Tomando como base este escenario de trabajo proponen un marco de recomendación inducido por la actividad y el comportamiento de los usuarios, para sugerir puntos de interés. Este marco incluye un módulo de perfilamiento del usuario que combina minería de preferencias contextuales y minería de sentimientos, para posteriormente realizar agrupamiento de dichos perfiles. Los clústeres obtenidos son posteriormente combinados con los perfiles individuales de los usuarios, para una mejor representación de estos. En un segundo momento, el módulo de recomendación de puntos de interés utiliza pre-filtrado de ítems acorde a los requerimientos del perfil de usuario correspondiente, para posteriormente realizar un proceso de ranking de puntos de interés, que considera métodos de recomendación basados en vecindario y relaciones de confianza entre los usuarios.

En adición, presenta un modelo de recomendación grupal que utiliza las relaciones sociales entre los usuarios para obtener ponderaciones que son utilizadas en reubicar, siguiendo un proceso de re-ranking, el listado de recomendaciones que se obtiene por el primer modelo.

Estas propuestas se evalúan en bases de datos obtenidas de plataformas reconocidas dentro del ámbito del turismo, como Yelp y TripAdvisor. Finalmente, se desarrolló un sistema recomendador individual y grupal, para implementar los desarrollos obtenidos. Este sistema, denominado XplorerVU TwB (Figura 4), permite a los usuarios especificar información en términos de tipo de recomendación deseada (individual o grupal), el tipo de actividad que se desea (aventura, naturaleza, eventos, vida nocturna, etc), el nivel de sorpresa y de energía asociado a la recomendación, así como la posibilidad de compartir la localización actual.
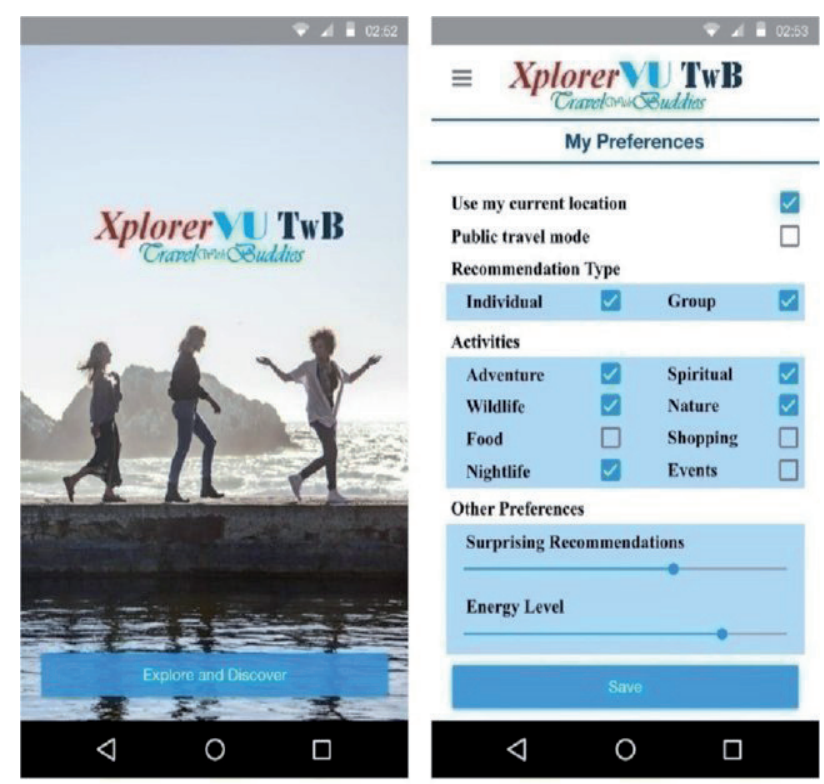

Figura 4: Interfaz del sistema XplorerVU TwB.

\section{Sistema enfocado a mejorar la experiencia del usuario en proceso de post-recomendación}

Alves et al [42] introduce un modelo conceptual enfocado en mejorar la experiencia del turista en un sistema recomendador grupal, específicamente para el proceso post-recomendación. Con este fin se utilizan técnicas de gamificación para adquirir el perfil del turista [43], agentes inteligentes modelados con los perfiles y los contextos de los turistas que incluyen aspectos psicológicos y socio-culturales, y diálogos entre los agentes basados en argumentación.

Específicamente, la arquitectura que se presenta está compuesta por diferentes servicios. El servicio multiagente, responsable de modelar los agentes inteligentes acorde a la información del turista (perfil y contexto) y otros agentes necesarios para el proceso; el servicio de recomendación, que se encarga de iterativamente generar las recomendaciones tanto individuales como grupales basadas en toda la información disponible (perfiles, contexto, etc); el servicio de puntos de interés que es obtenido desde Google Maps o aplicaciones similares y que se integra 
dentro del módulo de recomendación; el servicio de red social que permite incorporar nueva información de interés para la recomendación, y el módulo de gamificación que pretende favorecer la recopilación de información por parte de los turistas.

Como conclusión del trabajo, se afirma que, aunque el objetivo del mismo no es enfocarse en la propuesta de un nuevo algoritmo para la generación de un listado de recomendaciones, se espera que este facilite el consenso por parte de los usuarios en la etapa de post-recomendación, permitiendo la elección de opciones de mayor calidad y con una mayor satisfacción.

\section{Discusión}

La presente sección se centra en realizar un análisis de los trabajos previamente analizados, incluyendo una comparación crítica de los mismos y la discusión de líneas futuras a las que estos dan lugar.

\section{Comparación entre los trabajos identificados}

La tabla 1 presenta una comparación entre los trabajos analizados, atendiendo a diferentes criterios. Se refleja el tipo de estudio en el que mayormente se centra el trabajo (en la propuesta de un nuevo método o metodología, o si es mayormente la propuesta de nuevo software); el tipo de evaluación en el sentido de que si es usando bases de datos de referencia concebidas con este fin, o un estudio de usuarios [44]; y el escenario de aplicación de la propuesta. Asimismo, se reflejan las principales fortalezas y debilida- des asociadas a cada trabajo y el país en que mayormente se desarrolla la investigación.

La tabla muestra claramente la existencia de dos tipos de trabajos fundamentales. Estos son por una parte el desarrollo de plataformas de software y arquitecturas en las que se integran métodos de recomendación previamente desarrollados $[27,40,42]$, que se evalúan a través de estudios de usuarios o en los que no se realiza evaluación.

Por otra parte, se identificaron algunos trabajos en los que se proponen nuevos métodos o algoritmos [34, 36, 37, $39,41]$, pero en los que en muchos casos la evaluación de los mismos resulta incompleta, al centrarse en bases de datos artificiales [34], o limitándose a la realización de estudios de usuarios [37]. No se reporta un uso extensivo de la evaluación offline como paso previo al desarrollo de estudios de usuario y evaluación online, como lo sugieren los protocolos establecidos en el área de los sistemas recomendadores [44, 45].

Con respecto al dominio de aplicación, se observa una convergencia por parte de las propuestas a considerar dominios como el de las rutas turísticas, o los puntos de interés. Estos trabajos tuvieron un mayor o menor nivel de ajuste y dependencia del dominio en cuestión, registrándose trabajos de propósito general dentro del dominio del turismo como De Pessemier et al. [37], o trabajos bien enfocados en un dominio específico como el de los puntos de interés [39]. En adición se identificaron trabajos con metodologías para cualquier dominio de recomendación, que posteriormente se aplican en un sistema informático para el turismo [36].

Finalmente, es de resaltar como este problema de

Tabla 1: Comparación entre los trabajos analizados.

\begin{tabular}{|c|c|c|c|c|c|c|}
\hline & Tipo de estudio & Tipo de evaluación & $\begin{array}{l}\text { Escenario de } \\
\text { aplicación }\end{array}$ & Fortalezas & Debilidades & País \\
\hline Christensen et al. [34] & $\begin{array}{c}\text { Nueva metodología } \\
\text { / algoritmo }\end{array}$ & $\begin{array}{l}\text { Usando datos } \\
\text { artificiales }\end{array}$ & Rutas turísticas & $\begin{array}{l}\text { Investigación de ciclo } \\
\text { completo que incluye } \\
\text { nueva metodología y } \\
\text { desarrollo de sistema }\end{array}$ & $\begin{array}{l}\text { Explotación aún } \\
\text { insuficiente de las } \\
\text { relaciones sociales, } \\
\text { lo que constituye el } \\
\text { núcleo del trabajo }\end{array}$ & Argentina \\
\hline Wang et al. [36] & $\begin{array}{c}\text { Nueva metodología } \\
\text { / algoritmo }\end{array}$ & $\begin{array}{l}\text { Usando bases de } \\
\text { datos de referencia } \\
\text { internacional }\end{array}$ & Actividades turísticas & $\begin{array}{c}\text { Uso de técnicas } \\
\text { avanzadas como } \\
\text { factorización matricial }\end{array}$ & $\begin{array}{c}\text { Escasamente } \\
\text { centrado en el } \\
\text { dominio del turismo }\end{array}$ & Australia \\
\hline De Pessemier et al. [37] & $\begin{array}{l}\text { Nueva metodología/ } \\
\text { algoritmo }\end{array}$ & Estudio de usuarios & Destinos turísticos & $\begin{array}{c}\text { El uso de } \\
\text { recomendación basada } \\
\text { en restricciones }\end{array}$ & \begin{tabular}{|c|} 
No se realiza \\
experimentación con \\
valores de preferencias
\end{tabular} & Bélgica \\
\hline Nguyen y Ricci [27] & $\begin{array}{l}\text { Nueva plataforma } \\
\text { / arquitectura }\end{array}$ & Estudio de usuarios & Puntos de interés & $\begin{array}{c}\text { La adecuada } \\
\text { incorporación de } \\
\text { recomendación basada } \\
\text { en contenido, en el } \\
\text { contexto de un chat } \\
\end{array}$ & $\begin{array}{c}\text { Insuficiente evaluación } \\
\text { con vistas a medir } \\
\text { la efectividad de las } \\
\text { recomendaciones } \\
\text { generadas } \\
\end{array}$ & Italia \\
\hline Herzog et al. [39] & $\begin{array}{l}\text { Nueva metodología } \\
\text { / algoritmo }\end{array}$ & Estudio de usuarios & Puntos de interés & \begin{tabular}{|c|} 
El uso de funciones \\
de agregación \\
novedosas, ajustadas \\
al dominio en cuestión \\
(puntos de interés) \\
\end{tabular} & $\begin{array}{c}\text { Propuesta no utilizable } \\
\text { en otros dominios } \\
\text { de recomendación }\end{array}$ & Alemania \\
\hline Álvarez y Ziegler [40] & $\begin{array}{l}\text { Nueva plataforma } \\
\text { / arquitectura }\end{array}$ & Estudio de usuarios & Hoteles & $\begin{array}{c}\text { Considera cuestiones } \\
\text { novedosas tales } \\
\text { como negociación } \\
\text { y reconciliación de } \\
\text { preferencias. }\end{array}$ & $\begin{array}{c}\text { No se realizan estudios } \\
\text { de eficacia de la } \\
\text { recomendación }\end{array}$ & Alemania \\
\hline Longesh et al. [41] & $\begin{array}{l}\text { Nueva metodología } \\
\text { / algoritmo }\end{array}$ & $\begin{array}{l}\text { Usando bases de } \\
\text { datos de referencia } \\
\text { internacional }\end{array}$ & Puntos de interés & $\begin{array}{l}\text { El uso de fuentes de } \\
\text { datos reconocidas } \\
\text { como TripAdvisor }\end{array}$ & $\begin{array}{c}\text { Descripción macro de la } \\
\text { propuesta, que dificulta } \\
\text { reproductibilidad }\end{array}$ & India \\
\hline Alves et al [42] & $\begin{array}{l}\text { Nueva plataforma } \\
\text { / arquitectura }\end{array}$ & $\begin{array}{l}\text { No se realiza } \\
\text { evaluación. (Trabajo } \\
\text { en desarrollo) }\end{array}$ & $\begin{array}{l}\text { Ítems en general, en el } \\
\text { dominio del turismo }\end{array}$ & $\begin{array}{l}\text { Elementos novedosos } \\
\text { como el uso de la } \\
\text { gamificación }\end{array}$ & $\begin{array}{c}\text { Descripción muy macro } \\
\text { de la propuesta }\end{array}$ & Portugal \\
\hline
\end{tabular}


investigación ha sido previamente abordado por autores de diferentes países y culturas, tales como Argentina, Italia, Bélgica, Alemania, India, Australia, entre otros.

\section{Líneas futuras a desarrollar}

El análisis realizado a lo largo de este trabajo detectó que en los últimos años ha ocurrido un incremento del desarrollo de estudios de usuarios, restándole protagonismo al desarrollo de investigaciones de ciclo completo que incluyan la construcción de nuevos algoritmos y metodologías que concluyan con un extenso estudio experimental, centrados en el dominio de la recomendación grupal para el turismo. Así, resulta necesario el desarrollo de nuevas propuestas centradas en esta dirección, que puedan ser directamente reutilizadas por otros desarrolladores de sistemas informáticos enfocados en el dominio del turismo.

Más específicamente, es preciso la construcción de nuevas propuestas que profundicen en nuevas técnicas para agregar preferencias individuales en el nivel de grupo, en cómo distinguir entre grupos satisfechos y no satisfechos, cómo correlacionar y comparar las preferencias individuales con las grupales, y cómo relacionar las preferencias personales como la personalidad de los usuarios, tal y como recientemente también lo han reflejado otros autores como Delic et al. [46].

Se hace necesario también utilizar de una forma más activa los paradigmas tradicionales de recomendación, como la recomendación basada en contenido y el filtrado colaborativo [19], y no considerar la solución al problema abordado en el presente trabajo únicamente a través de meras extensiones de los sistemas recomendadores individuales. Tomando en cuenta los trabajos analizados, únicamente Wang et al. [36] y Herzog et al. [39] realizan incipientes esfuerzos en este sentido. Es necesario también considerar otros criterios de calidad de las recomendaciones, como diversidad [47] y cubrimiento [35] del listado del listado de recomendaciones generadas.

Finalmente, resulta necesario explorar nuevos dominios de recomendación dentro de la propia área del turismo, tal como la recomendación de secuencias de actividades de innovación, inicialmente propuesto por Carballo-Cruz et al. $[48,49]$.

\section{Conclusiones}

El presente trabajo se centró en realizar una revisión del rol de los sistemas recomendadores grupales como herramienta innovadora en el área del turismo. Con este fin, se desarrolló un análisis de los trabajos recientes más relevantes identificados en la literatura, centrados en el problema de la recomendación grupal de ítems asociados al dominio del turismo.

El análisis arrojó como resultados principales la presencia de una convergencia de dominios de aplicación de los sistemas recomendadores grupales en el área del turismo, para considerar dominios como el de las rutas turísticas o los puntos de interés. No obstante, no se identificó el uso extensivo de la evaluación offline como paso inicial del proceso de evaluación, como lo sugieren los protocolos establecidos en el área de los sistemas recomendadores [44, 45]. A raíz de esto, se consideró insuficiente el desarrollo investigativo centrado en la propuesta de nuevos métodos y algoritmos centrados en este problema en particular.

Estas conclusiones dan lugar por tanto a la propuesta de líneas futuras a explorar, entre las que se mencionan el desarrollo de nuevas propuestas centradas en nuevos algoritmos y metodologías que concluyan con un extenso estudio experimental, que puedan ser directamente reutilizadas por otros desarrolladores de sistemas informáticos enfocados en el dominio del turismo. Asimismo, se necesita considerar la creación de nuevas propuestas que tomen en cuenta otros criterios de calidad de las recomendaciones, como la diversidad [47] y el cubrimiento [35].

Esperamos que el presente trabajo sea útil para la comunidad científica, con vistas a continuar desarrollando la todavía incipiente línea de investigación de sistemas recomendadores grupales para el turismo.

\section{Referencias Bibliográficas}

1. A. Bellogín, et al., "An empirical comparison of social, collaborative filtering, and hybrid recommenders, ” ACM Transactions on Intelligent Systems and Technology (TIST), vol. 4, p. 14, 2013.

2. D. Bernardes, et al., "A Social Formalism and Survey for Recommender Systems, ” ACM SIGKDD Explorations Newsletter, vol. 16, pp. 20-37, 2015.

3. J. Bobadilla, et al., "Recommender systems survey," Knowledge-Based Systems, vol. 46, pp. 109-132, 2013.

4. L. Lü, et al., "Recommender systems," Physics Reports, vol. 519, pp. 1-49, 2012.

5. s. Dara, et al., "A survey on group recommender systems, ” Journal of Intelligent Information Systems, vol. 54, pp. 271-295, 2020.

6. J. Castro, et al., "An empirical study of natural noise management in group recommendation systems, ” Decision Support Systems, vol. 94, pp. 1-11, 2017.

7. z. Huang, et al., "A comparison of collaborative-filtering recommendation algorithms for e-commerce, " IEEE Intelligent Systems, pp. 68-78, 2007.

8. R. Yera and L. Martínez, "A recommendation approach for programming online judges supported by data preprocessing techniques, ”Applied Intelligence, vol. 47, pp. 277-290, 2017.

9. R. Yera Toledo, et al., "A recommender system for programming online judges using fuzzy information modeling," in Informatics, 2018, p. 17.

10. J. Lu, et al., "Recommender system application develop- 
ments: A survey, ” Decision Support Systems, vol. 74, pp. 12-32, 2015.

11. M. Li, et al., "An approach to task-oriented knowledge recommendation based on multi-granularity fuzzy linguistic method," Kybernetes, vol. 44, pp. 460-474, 2015.

12. J. N. Cappella, et al., "Constructing Recommendation Systems for Effective Health Messages Using Content, Collaborative, and Hybrid Algorithms, "The ANNALS of the American Academy of Political and Social Science, vol. 659, pp. 290-306, 2015.

13. R. Yera, et al., "A Food Recommender System Considering Nutritional Information and User Preferences," IEEE Access, vol. 7, pp. 96695-96711, 2019.

14. D.-Y. Yeh and C.-H. Cheng, "Recommendation system for popular tourist attractions in Taiwan using Delphi panel and repertory grid techniques, " Tourism Management, vol. 46, pp. 164-176, 2015.

15. E. Carballo-cruz, et al., "Del desarrollo de capacidades de aprendizaje a la satisfacción del cliente en una instalación hotelera, ”Investigaciones Turísticas, vol. 2, pp. 82-101, 2011.

16. S. Renjith, et al., "An extensive study on the evolution of context-aware personalized travel recommender systems, ”Information Processing \& Management, vol. 57, p. 102078, 2020.

17. K. Chaudhari and A. Thakkar, "A Comprehensive Survey on Travel Recommender Systems, ” Archives of Computational Methods in Engineering, vol. 27, pp. 1545-1571, 2020.

18. P. Lops, et al., "Content-based recommender systems: state of the art and trends " in Recommender Systems Handbook, F. Ricci, et al., Eds., ed New York: Springer, 2011, pp. 73-100.

19. G. Adomavicius and A. Tuzhilin, "Toward the next generation of recommender systems: A survey of the state-ofthe-art and possible extensions, " IEEE Transactions on Knowledge and Data Engineering, vol. 17, pp. 734749, 2005.

20. R. Yera, et al., "A Regularity-Based Preprocessing Method for Collaborative Recommender Systems, ” Journal of Information Processing Systems, vol. 9, pp. 435-460, 2013.

21. M. J. Pazzani, "A framework for collaborative, contentbased, and demographic filtering., ” Artificial Intelligence Review, vol. 13, pp. 393-408, 1999.

22. C. Porcel, et al., "A hybrid recommender system for the selective dissemination of research resources in a technology transfer office, " Information Sciences, vol. 184, pp. 1-19, 2012.

23. R. Burke, "Hybrid recommender systems:survey and experiments, "User Modeling and User-Adapted Interaction, vol. 12, pp. 331-370, 2002.

24. M. G. Vozalis and K. G. Margaritis, "Using SVD and demographic data for the enhancement of generalized co- llaborative filtering, "' Information Sciences, vol. 177, pp. 3017-3037, 2007.

25. K. Choi, et al., "A hybrid online-product recommendation system: Combining implicit rating-based collaborative filtering and sequential pattern analysis, "Electronic Commerce Research and Applications, vol. 11, pp. 309-317, 2012.

26. $x$. Su, et al., "Hybrid collaborative filtering algorithms using a mixture of experts, " in Proceedings of the IEEE/WIC/ACM International Conference on Web Intelligence (WI '07), Silicon Valley, Calif, USA, 2007, pp. 645-649.

27. T. N. Nguyen and F. Ricci, "A chat-based group recommender system for tourism, " Information Technology \& Tourism, vol. 18, pp. 5-28, 2018.

28. L. Sun, et al., "Applying uncertainty theory into the restaurant recommender system based on sentiment analysis of online Chinese reviews, "World Wide Web, vol. 22, pp. 83-100, 2019.

29. R. Hti and M. S. Desarkar, "Personalized tourist package recommendation using graph based approach, " in Adjunct Publication of the 26th Conference on User Modeling, Adaptation and Personalization, 2018, pp. 257-262.

30. G. Pavlidis, "Recommender systems, cultural heritage applications, and the way forward, ”Journal of Cultural Heritage, vol. 35, pp. 183-196, 2019.

31. T. De Pessemier, et al., "Comparison of group recommendation algorithms, " Multimedia tools and applications, vol. 72, pp. 2497-2541, 2014.

32. R. Yera and L. Martínez, "Fuzzy tools in recommender systems: A survey, " International Journal of Computational Intelligence Systems, vol. 10, pp. 776-803, 2017.

33. M. Kompan and M. Bielikova, "Group recommendations: Survey and perspectives, "Computing and Informatics, vol. 33, pp. 1-31, 2014.

34. I. Christensen, et al., "Social group recommendation in the tourism domain, " Journal of Intelligent Information Systems, vol. 47, pp. 209-231, 2016.

35. A. Gunawardana and G. Shani, "A Survey of Accuracy Evaluation Metrics of Recommendation Tasks, ” Journal of Machine Learning Research, vol. 10, pp. 2935-2962, 2009.

36. W. Wang, et al., "Member contribution-based group recommender system, ” Decision Support Systems, vol. 87, pp. 80-93, 2016.

37. T. De Pessemier, et al., "Hybrid group recommendations for a travel service," Multimedia tools and applications, vol. 76, pp. 2787-2811, 2017.

38. P. Favardin, et al., "Borda rule, Copeland method and strategic manipulation, " Review of Economic Design, vol. 7, pp. 213-228, 2002.

39. D. Herzog, et al., "TourRec - A Tourist Trip Recommender System for Individuals and Groups, " in Proceedings of the 12th ACM Conference on Recommender 
Systems, 2018, pp. 496-497.

40. J. Álvarez and J. Ziegler, "Negotiation and Reconciliation of Preferences in a Group Recommender System," Journal of Information Processing, vol. 26, pp. 186-200, 2018.

41. R. Logesh, et al., "Efficient user profiling based intelligent travel recommender system for individual and group of users, "Mobile Networks and Applications, vol. 24, pp. 1018-1033, 2019.

42. P. Alves, et al., "Modeling a Mobile Group Recommender System for Tourism with Intelligent Agents and Gamification, " in International Conference on Hybrid Artificial Intelligence Systems, 2019, pp. 577-588.

43. K. Seaborn and D. I. Fels, "Gamification in theory and action: A survey, " International Journal of human-computer studies, vol. 74, pp. 14-31, 2015.

44. F. Ricci, et al., Recommender Systems Handbook 2nd Edition. New York: Springer, 2015.

45. G. Shani and A. Gunawardana, "Tutorial on applicationoriented evaluation of recommendation systems, " AI Communications, vol. 26, pp. 225-236, 2013.
46. A. Delic, et al., "An observational user study for group recommender systems in the tourism domain," Information Technology \& Tourism, vol. 19, pp. 87-116, 2018.

47. A. Javari and M. Jalili, "A probabilistic model to resolve diversity-accuracy challenge of recommendation systems, " Knowledge and Information Systems, vol. 44, pp. 609-627, 2015.

48. E. Carballo-cruz, et al., "An Intelligent System for Sequencing Product Innovation Activities in Hotels, " IEEE Latin America Transactions, vol. 17, pp. 305-315, 2019.

49. E. Carballo-Cruz, et al., "La innovación de producto en la formación de la imagen percibida. Caso Hotel Colonial Cayo Coco, destino turístico Jardines del Rey, Cuba," Retos de la Dirección, vol. 10, pp. 114-141, 2016. 